\title{
Proposal for a Method of Non-restrictive Measurement of Resting Heart Rate in a Lying Position
}

\author{
Shima Okada ${ }^{1) *}$, Yoshihisa Fujiwara ${ }^{1)}$, Shingo Suzuki ${ }^{2}$, Masashi Yasuda ${ }^{1)}$, Masaaki Makikawa ${ }^{3)}$ \\ and Takeo Iida ${ }^{3)}$ \\ 1) Ecology and Energy Systems Development Center, SANYO Electric Co., Ltd. \\ 2) Graduate School of Science and Engineering, Ritsumeikan University \\ 3) College of Science and Engineering, Ritsumeikan University
}

\begin{abstract}
Daily monitoring of heart rates is important in health management. Many researchers have analysed heart rate variability by using the resting heart rate because such an analysis can facilitate the early discovery of a variety of illnesses and health conditions.

Some problems that arise in measuring heart rate are the feeling of confinement. Therefore, we required a system that could measure the resting heart rate in a static position in such a way that the subject is completely unaware that the measurement is being recorded. We propose a non-restrictive measurement method that uses only an acceleration sensor placed inside a down quilt. This method is easy for home use.

The acceleration sensor was placed inside the quilt such that it was positioned opposite to the left-hand side of the subject's chest. Six healthy subjects were requested to lie in the supine position and were covered with the quilt equipped with the acceleration sensor. Mechanical vibrations that resulted from heart activity were carried through the quilt to the acceleration sensor.

As a result, periodic vibrations were measured successfully, and in the six subjects, these vibrations were proved to be highly correlated with the $\mathrm{R}$ wave of electrocardiograms. The same results were obtained even when the subjects were lying in the left lateral position. The results indicated that our new method, which used an acceleration sensor placed inside a down quilt, was simple and could be used to measure the resting heart rate in a lying position. $J$ Physiol Anthropol 25(4): 299-305, $2006 \mathrm{http} / / / w w w . j s t a g e . j s t . g o . j p / b r o w s e / j p a 2$

[DOI: 10.2114/jpa2.25.299]
\end{abstract}

Keywords: resting heart rate, acceleration sensor, nonrestrictive measurement, lying position

\section{Introduction}

Heart rate (HR) monitoring is important in health management. For example, high blood pressure (Guzzetti et al., 1988; Mandawat et al., 1995; Kohara et al., 1995), diabetes (Wheeler and Watkins, 1973), and obesity (Kupari et al., 1993) are accompanied by a decrease in HR variability, while psychological stress (Kamada, 1992), lack of exercise (Goldsmith et al., 1992), and other factors are also known to affect HR variability. Research has also been conducted in relation to the prognosis of heart diseases, particularly that following myocardial infarction (Keiger et al., 1987; Bigger et al., 1989). In these types of studies, researchers analysed HR variability in many cases by using the resting HR because the resting HR variability can serve as an index of sympathetic or parasympathetic dominance based on studies of the frequency of HR variability (Sharpley et al., 2000). For predicting the prognosis of such lifestyle-related diseases, we should measure the resting HR in the non-restrictive method.

A number of methods have been previously proposed for non-restrictive measurement of the resting HR in a lying position. Along with these, detection of the resting HR and respiration by measuring changes in electrical capacitance has also been reported (Allihanka et al., 1988). In recent years, many new sensors that offer comparatively easy operation and installation have been developed. Such methods include nonrestrictive measurements of respiration and HRs from the internal pressure changes in an air mat that is affected by body motion due to respiration or heart activity (Watanabe and Watanabe, 1999). They also include non-restrictive measurements of respiration and HRs using sheets or pillows with a wire-shaped respiratory pickup sensor attached to an air mat (Tanaka, 2000). However, these methods present a specific problem known as 'ballistocardiography' (Tomek, 1975).

* Affiliation since April 2006: Osaka University Graduate School of Medicine, Division of Health Science. 
a)

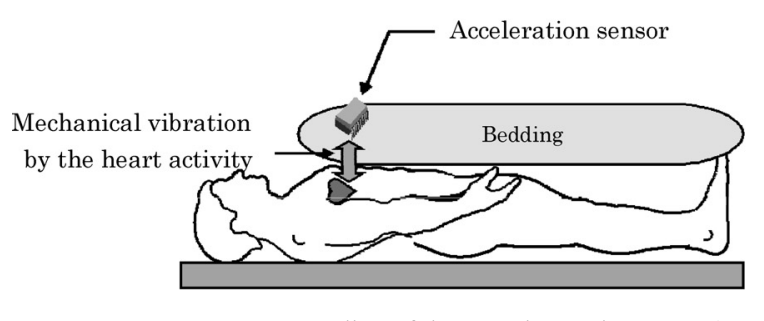

b)

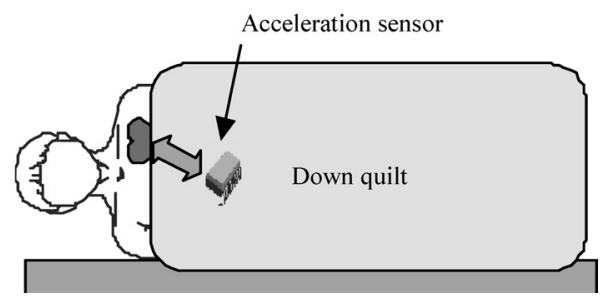

Fig. 1 Outline of the experimental system, a) Supine position; b) Left lateral position.

Ballistocardiography is a method used for measuring fine body motions caused by heart activity and blood movement in blood vessels. The basic problem with this method is that it is influenced by the body weight, movement of organs inside the abdomen, and distortion of fat and musculature.

Based on this, we require a device that can measure the resting HR in a static position in such a way that the subject is completely unaware that the measurement is being recorded. In this study, we proposed a new method for non-restrictive measurement of the resting HR in a lying position using an acceleration sensor placed inside a down quilt and confirmed the accuracy of the method.

\section{Methods}

\section{Subjects}

A total of six healthy males with a mean $( \pm \mathrm{SD})$ age of $22.4 \pm 1.5$ years, mean height of $171.4 \pm 5.9 \mathrm{~cm}$, and mean weight of $61 \pm 7.7 \mathrm{~kg}$ agreed to participate in tests designed to evaluate the performance of the proposed method. They did not have any pre-existing heart disease. After we fully explained the purpose of the experiment, the procedure, and the potential risk and ensured that they understood this information completely, the subjects agreed to participate.

\section{Experimental system}

We present the experimental system used for non-restrictive measurement of the resting HR in a lying position. Figure 1a) shows an outline of the experimental system. A small acceleration sensor is installed in the middle layer of a down quilt. When the subject lies down and is covered with the quilt, the minute mechanical vibrations resulting from the subject's heart activity are transmitted to the quilt where they are picked up by the acceleration sensor. The acceleration sensor is sewn into the quilt directly above the left side of the anterior chest, where the heart is located. This system employs a piezoelectric acceleration sensor and was set with its sensitivity axis (z-axis) oriented to earth's gravity.

The subject's lying position may adversely affect nonrestrictive measurement of the resting HR; however, as discussed later, no problems should occur if the subject is in a lateral position. As shown in Fig. 1b), the sensitivity axis of

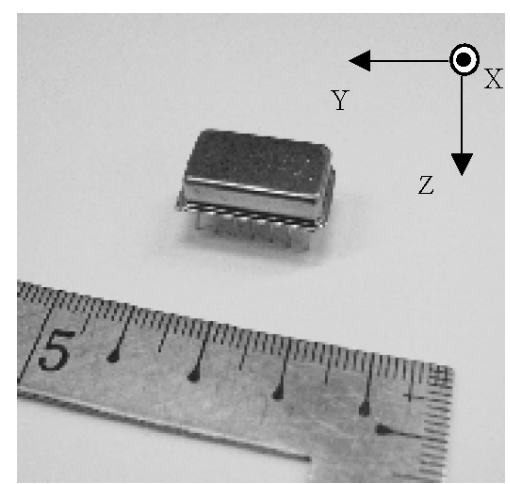

Fig. 2 Picture of the acceleration sensor.

Table 1 Characteristics of the acceleration sensor

\begin{tabular}{ll}
\hline \multicolumn{1}{c}{ Data item } & \multicolumn{1}{c}{ Value } \\
\hline Range of detection & $\pm 1\left(\mathrm{~ms}^{-2}\right)$ \\
Sensitivity of detection & $1.9\left(\mathrm{~V} / \mathrm{ms}^{-2}\right)$ \\
Response frequency & $0.8 \sim 1000(\mathrm{~Hz})$ \\
Resonance frequency & $10 \sim 15(\mathrm{kHz})$ \\
Size & $\mathrm{W} 20.5^{*} \mathrm{D} 12.5^{*} \mathrm{H} 5(\mathrm{~mm})$ \\
Electrical requirements & $0.5(\mathrm{~mA}) 5(\mathrm{~V})$ \\
Weight & $4(\mathrm{~g})$ \\
\hline
\end{tabular}

the acceleration sensor installed in the quilt is always perpendicular to the surface of the quilt; therefore, this axis still faces the heart when the subject is in a lateral position. As a result, the sensitivity of the measurements obtained by using this experimental system depends more on the distance from the heart to the sensor than on the subject's position.

The picture of acceleration sensor is shown in Fig. 2. Table 1 shows the specifications of the piezoelectric acceleration sensor (MA3-01Aa; Micro Stone Co., Ltd.,). The sensor has a built-in amplifier and its output has a comparatively highsensitivity of $1.9 \mathrm{~V}$ per $\mathrm{ms}^{-2}$ of acceleration. This sensor also shows high linearity and a response frequency of more than $0.8 \mathrm{~Hz}$; therefore, it prevents overlapping of the vibrations from heart activity with those from respiration. Furthermore, the sensor is small and light, so even when it is installed in the quilt, it does not cause discomfort to the subject. Additionally, the low consumption current of $0.5 \mathrm{~mA}$ and voltage of $5 \mathrm{~V}$ 
allows for measurements under battery power over a long time period. The original resonant frequency is between 10 and $15 \mathrm{kHz}$, which is sufficiently high in comparison to the frequency of mechanical vibrations generated by heart activity. Data on heart activity was obtained with this acceleration sensor at $200 \mathrm{~Hz}$ using a 12-bit A/D conversion board (PCI3153, Interface Corp.), and it was stored in a PC for further analysis.

\section{Experimental procedure}

The measurements were performed at Ritsumeikan University. To evaluate this method, the resting HR was obtained by the system described above and by ECG measurements for the same period of time.

In these experiments, the subjects were asked to lie quietly on their backs and were then covered with the quilt equipped with the acceleration sensor. The resting HRs of the subjects were measured for 5 minutes after the 5-minute rest period. The subject first lay down in the supine position and then in the left lateral position. At this time, the changing position was limited as directed by the experimenter.

Next, this method was used to measure the heart activity of one subject throughout the course of one night. This was performed based on the assumption that in our daily lives, the most common state in a lying position is the sleeping state. The time period of the measurements was 6 hours and 45 minutes.

\section{Results and Discussion}

\section{Measurements in the supine position}

Figure 3 shows an example of the use of our system and ECG for detecting the HR signal in the supine position for 30 seconds in subject N.S. As shown in this graph, high-frequency noise overlapped the HR signal generated by the experimental system; however, the peak was obtained at the same position as the ECG-R wave. Furthermore, the peak value was sufficiently large (maximum acceleration: $0.06 \mathrm{~ms}^{-2}$ ) to be detected automatically by using the threshold scheme. The results demonstrated that the proposed HR measurement method was effective. Table 2 shows the results of 5-minute measurements on the six subjects, where ' $\mathrm{N}$ ' is the number of the HR peak detected by the experimental system over a period of 5 minutes. 'Mean', 'Max.', and 'Min.' are the mean, maximum, and minimum peak amplitudes. With respect to 'Correlation', we calculated the R-R interval for each beat of the heart and scored the correlation coefficient between the data obtained from ECGs and those obtained from the acceleration sensor over a period of 5 minutes. In addition, Table 2 shows the measurement accuracy of 5-minute measurements on the six subjects, where $\mathrm{N}$ is the detected $\mathrm{R}$ wave peaks and 'Max. Error' is the maximum difference time between the R-R interval from ECG and that from acceleration. 'SEM' represents the standard error of the mean difference between the R-R interval from ECG and that from acceleration.

As shown in this table, we were able to obtain resting HR measurements for all subjects. When mean accelerations were compared, the largest difference in the amplitude of acceleration was $0.02 \mathrm{~ms}^{-2}$ in the case of subjects Y.H. and K.Y. A possible reason for this difference was the decrease in acceleration resulting from the contact condition of the sensor. The average correlation coefficient for the six subjects was 0.98 .

We then showed the measurement accuracy in detail. For each of the two methods, one result of the R-R interval time over a period of 5 minutes is shown in Fig. 4. This figure shows the result of subject N.S., whose correlation coefficient was 0.99 in Table 2. We were able to confirm that the waves were similar in shape.

Max. Error and SEM values were very low. It was thought that the detection accuracy with the acceleration sensor in the supine position was similar to that obtained with ECG. This suggested that the R-R interval obtained from ECG and the

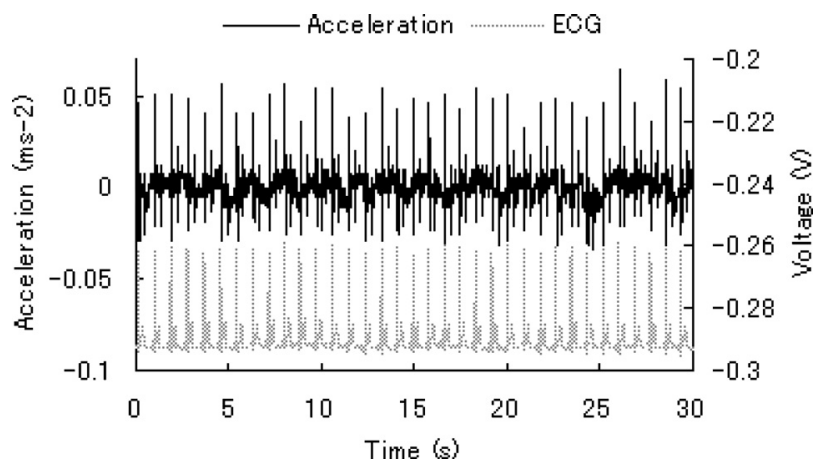

Fig. 3 Signals detected using the experimental system and ECG in subjects lying in the supine position.

Table 2 Results from the use of the experimental system to measure the heart rates of subjects lying in the supine position

\begin{tabular}{|c|c|c|c|c|c|c|c|}
\hline Subject & $\mathrm{N}$ & Mean $\left(\mathrm{ms}^{-2}\right)$ & $\operatorname{Max} .\left(\mathrm{ms}^{-2}\right)$ & Min. $\left(\mathrm{ms}^{-2}\right)$ & Correlation & Max. Error (ms) & SEM \\
\hline Y.A. & 345 & 0.04 & 0.07 & 0.02 & 0.99 & 0.01 & 0.0001 \\
\hline K.Y. & 323 & 0.04 & 0.06 & 0.02 & 0.96 & 0.08 & 0.0009 \\
\hline Y.H. & 337 & 0.05 & 0.08 & 0.03 & 0.99 & 0.01 & 0.0002 \\
\hline K.S. & 368 & 0.04 & 0.07 & 0.02 & 0.97 & 0.06 & 0.0004 \\
\hline K.N. & 320 & 0.04 & 0.07 & 0.03 & 0.98 & 0.08 & 0.0023 \\
\hline N.S. & 345 & 0.05 & 0.08 & 0.03 & 0.99 & 0.01 & 0.0002 \\
\hline Ave. & 340 & 0.04 & 0.07 & 0.03 & 0.98 & 0.04 & 0.0007 \\
\hline
\end{tabular}


data from the acceleration sensor were similar.

\section{Measurements in the lateral position}

To clarify the effects of position, the HR was measured with the subjects lying in the left lateral position. Figure 5 shows a 30 -second recording of subject N.S. with the proposed method and with ECG. The results showed that when the subject was

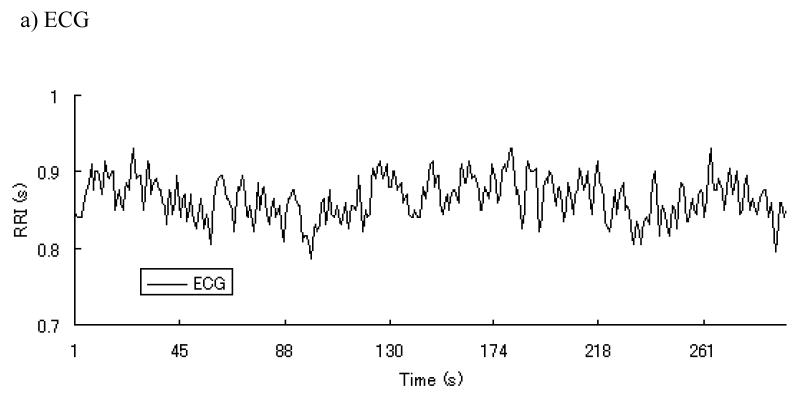

b) Acceleration

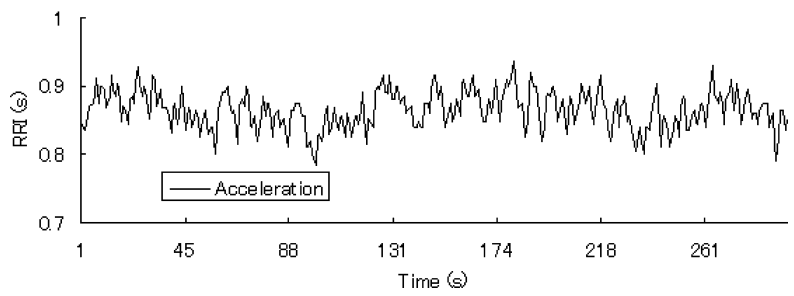

Fig. 4 Results of the R-R interval over a period of 5 minutes in subjects lying in the supine position a) ECG; b) Acceleration sensor.

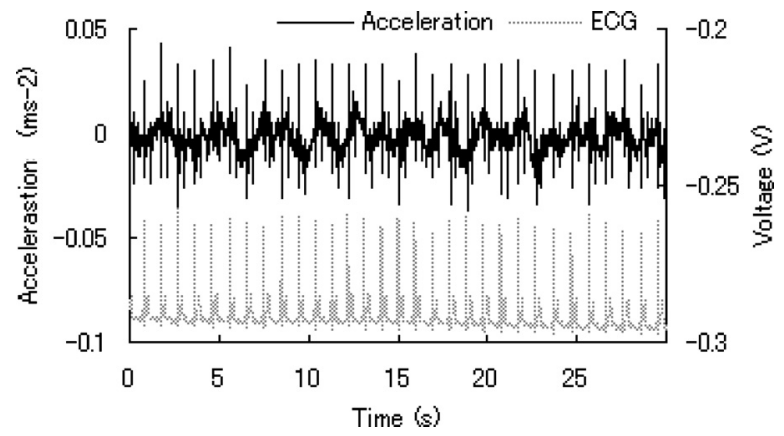

Fig. 5 Signals detected by the experimental system and ECG in subjects lying in the left lateral position. lying in the left lateral position, it was possible to distinguish the peaks in the same way as when the subject was in the supine position. Table 3 shows the results of the resting HR of the six subjects in the left lateral position, and data from subjects in the supine position were compared with those from subjects in the left lateral position. The method of calculating each value in the table was the same as that used for calculating the measurements shown in Table 2. The mean peak output value was $0.03 \mathrm{~ms}^{-2}$ in the left lateral position. This value was lower than the signal detected when the subject was in the supine position. With regard to the correlation coefficient, the average value of the six subjects was 0.93 ; this was 0.5 lower than the value obtained in the supine position. In comparison with the supine position, stable measurements of the HR could not be recorded in the left lateral position.

We next describe the measurement accuracy in detail. The R-R interval of subject K.S. over a period of five minutes is shown in Fig. 6. As shown in Table 3, his correlation coefficient was 0.86 , which was the lowest value among the six subjects. Figure 6 shows the result obtained with the acceleration sensor. It was similar to that obtained with ECG;

a)

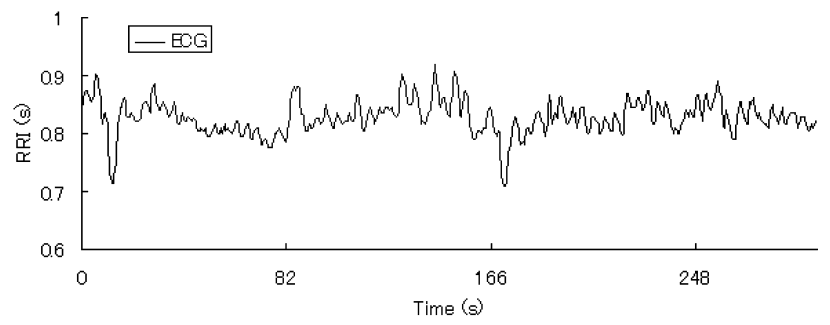

b)

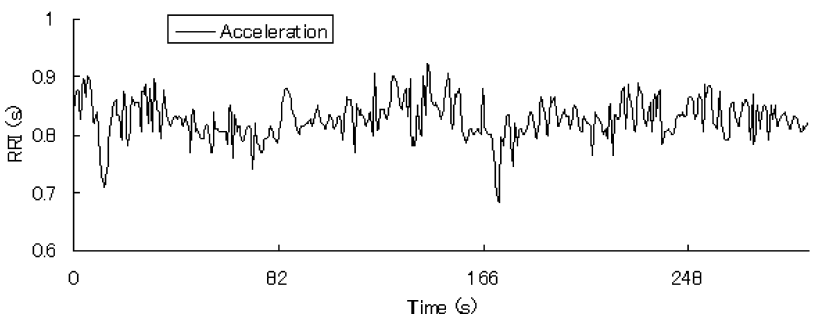

Fig. 6 Results of the R-R interval over a period of 5 minutes in subjects lying in the left lateral position. a) ECG; b) Acceleration sensor.

Table 3 Results from the use of the experimental system to measure the heart rates of subjects lying in the left lateral position

\begin{tabular}{|c|c|c|c|c|c|c|c|}
\hline Subject & $\mathrm{N}$ & Mean $\left(\mathrm{ms}^{-2}\right)$ & $\operatorname{Max} .\left(\mathrm{ms}^{-2}\right)$ & Min. $\left(\mathrm{ms}^{-2}\right)$ & Correlation & Max. Error (ms) & SEM \\
\hline Y.A. & 340 & 0.03 & 0.04 & 0.02 & 0.95 & 0.01 & 0.0004 \\
\hline K.Y. & 338 & 0.03 & 0.05 & 0.01 & 0.94 & 0.07 & 0.0155 \\
\hline Y.H. & 248 & 0.03 & 0.05 & 0.02 & 0.93 & 0.01 & 0.0036 \\
\hline K.S. & 419 & 0.03 & 0.05 & 0.02 & 0.86 & 0.07 & 0.0173 \\
\hline K.N. & 334 & 0.03 & 0.04 & 0.02 & 0.88 & 0.08 & 0.0233 \\
\hline N.S. & 326 & 0.05 & 0.07 & 0.03 & 0.99 & 0.01 & 0.0032 \\
\hline Ave. & 335 & 0.03 & 0.05 & 0.02 & 0.93 & 0.04 & 0.0105 \\
\hline
\end{tabular}


however, the values differed in some parts. SEM in the supine position was 0.0007 while that in the left lateral position was 0.0105 . Therefore, it was confirmed that both the correlation coefficient and the SEM in the left lateral position were lower than those in the supine position. Based on these results, it was considered that the $\mathrm{HR}$ in the left lateral position could obviously be detected; however, the accuracy was not as high as that of the measurement in the supine position.

\section{Measurement throughout the night}

This method was used in one subject to allow non-restrictive HR measurement throughout the course of one night. Figure 7 shows the original waveform from the acceleration sensor placed on subject N.S. throughout the night. We calculated the instantaneous HR during sleep, excluding regions that were clearly thought not to be related to HR. Figure 8 shows the results of HR validation obtained from the acceleration sensor and those of HRs obtained from ECGs measured at the same time on subject N.S. As shown in the diagram, variations are evident in the acceleration rate, similar to the instantaneous HRs calculated from ECGs.

Table 4 shows the variables of the HR for each hour during sleep. 'Number of samples' indicates the number of peaks detected. 'Data efficiency' refers to the value in which the number of peaks detected by the acceleration sensor is divided by the number detected by ECG. 'Correlation efficiency' shows the correlation between the R-R intervals detected by ECG and those detected by the acceleration sensor. 'Mean HR

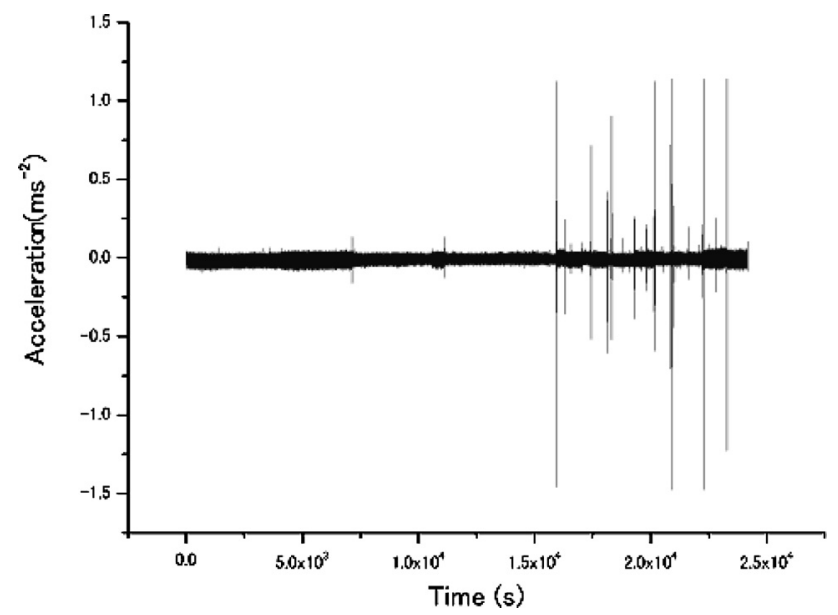

Fig. 7 Raw signals obtained by the acceleration sensor throughout the night.
(ECG)' is the mean HR obtained by ECG. 'Mean HR (barred time data)' shows the mean HRs calculated from the ECG in regions where peaks could not be detected by the acceleration sensor.

As shown in Table 4, it was not possible to measure heart activity over the entire sleeping period throughout the night. The data efficiency throughout the night ranged from $87.6 \% \sim 99.0 \%$, confirming that it was possible to measure the HR using this method. Even in the period from 5 to 6 hours,

a)

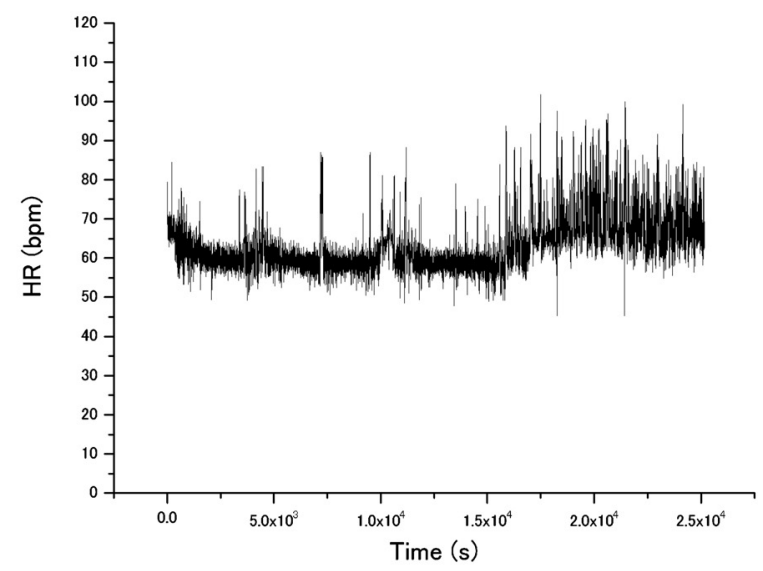

b)

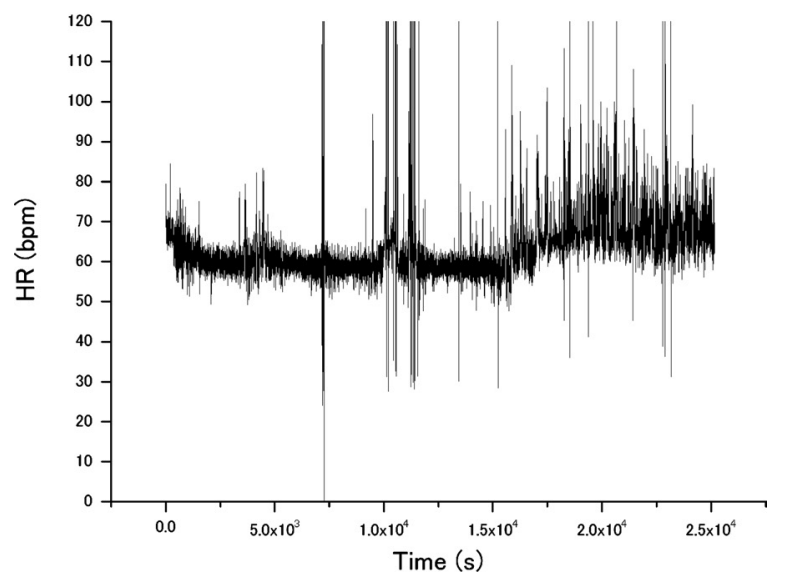

Fig. 8 HR results. a) ECG; b) Acceleration sensor throughout the night.

Table 4 Results from measurements of heart activity throughout the night

\begin{tabular}{lccccccc}
\hline \multicolumn{1}{c}{ Time (hours) } & 1 & 2 & 3 & 4 & 5 & 6 & $3 / 4$ \\
\hline Number of samples & 3,662 & 3,592 & 3,584 & 3,540 & 3,724 & 4,165 \\
Data efficiency (\%) & 99.0 & 98.4 & 96.6 & 94.5 & 95.1 & 87.6 & 94.4 \\
Correlation coefficients & 0.99 & 0.67 & 0.57 & 0.50 & 0.95 & 0.76 & 0.82 \\
Mean HR (ECG) (bpm) & 61.3 & 60.0 & 60.2 & 59.2 & 62.8 & 70.2 & 67.8 \\
Mean HR (barred time data) (bpm) & - & 77.3 & 77.6 & 69.3 & 83.3 & 82.1 \\
\hline
\end{tabular}


where maximum data was lost, the data efficiency was $87.6 \%$, confirming that heart activity can be detected.

Data efficiency was not $100 \%$ because large acceleration changes due to body movement contaminated the signal during sleep; consequently, the detection of heart activities at these times was impossible. Acceleration occasionally went out of range due to physical activities and sometimes decreased due to posture changes that occurred during sleep. Considering the mean instantaneous HRs calculated from the ECG in regions where peak detection was impossible, it was possible to observe increases in the HR. Given that the HR increases temporarily when the body moves during sleep (Nakajima and Osa, 1996), we could assume that such physical motion was a major cause of the deficiencies in the data. Furthermore, we could see that data efficiency decreases as sleeping time elapses. Earlier, we mentioned that physical activity was one of the causes of the inability to detect peaks; therefore, it was considered that body motion during sleep occurs frequently during the early sleeping period (Kripke et al., 1978). Given this situation, we could presume that data efficiency decreased because the number of physical motions increased as sleeping time elapsed.

With regard to the correlation coefficients presented in Table 4 , the difference of every time of correlation was large. Most correlation coefficients were 0.7 or higher, which indicated a strongly positive correlation; however, the occurrence of a time period where the correlation was low could be confirmed. At 4 hours, the correlation coefficient was the lowest-0.5. Although 0.5 appeared to represent a positive correlation, the accuracy was not sufficient for HR monitoring. As previously noted, the correlation coefficient tended to decrease in the left lateral position in comparison with the supine position. Therefore, we assumed that body movement with a change in the position resulted in a decrease in the value of the correlation coefficient. In addition, it might have resulted from a shift in the position of the acceleration sensor due to body movement or rolling over.

In order to resolve these problems, we considered the following methods. The present study was conducted with one acceleration sensor attached to the quilt. In this case, there was a higher chance that the acceleration sensor would shift due to body movement or rolling over. Therefore, we predict that the installation of several acceleration sensors will enable highly accurate measurements of the heart activity even when there is a great amount of physical activity.

\section{Conclusion}

In this study, we demonstrated that it was possible to measure the resting HR using a non-restrictive method, in which only an acceleration sensor is placed inside a down quilt. The advantage is that signal cables do not cause discomfort to the subject. In any case, this method allows a reduction in the psychological and physical burden imposed on the subject and achieves non-restrictive resting HR measurement over a long time period.

However, when this sensor was used throughout the night, we could not obtain high-accuracy short-term measurements of the HR. We plan to resolve this problem by placing several acceleration sensors in the quilt.

As noted in the Introduction, from the viewpoint of HR analysis in health management and maintenance, there is a growing need for a method that enables non-restrictive measurement of the resting HR. We consider that this method may be used in a number of other applications, including monitoring the HRs of the bedridden elderly and others confined to a bed over long time periods. Moreover, by linking this system to alarm systems, mobile phones, and other network terminals, it can also be used to assist in the monitoring of the elderly.

\section{References}

Allihanka J, Vashtoranta K, Saarikivi I (1988) A new method for long-term monitoring of the ballistocardiogram, heart rate, and respiration. Am J Physiol 240: 384-392

Bigger JT, Fleiss J, Steinman R, Rolnitzky LM, Kleiger RE, Rottoman JN (1992) Frequency domain measures of heart period variability and mortality after myocardial infarction. Circulation 85: 164-171

Echt DS, Liebson PR, Mitchell LB, Peters RW, Obias-Mannno D, Baker AH, Arensberg D, Baker A, Friedman L, Greene HL, Huther ML, Richardson DW (1991) Mortality and morbidity in patients receiving encainide, flecainide, or placebo. The Cardiac Arrhythmia Suppression Trial. N Engl J Med 324: 781-788

Goldsmith RL, Bigger JT Jr, Steinman RC, Fleiss JL (1992) Comparison of 24-hour parasympathetic activities in endurance-trained and untrained young men. J Am Coll Cardiol 20: 552-558

Guzzetti S, Casati R, Piccaluga E, Cerutti S, Lombardi F, Pagani M, Malliani A (1988) Sympathetic predominance in essential hypertension: a study employing spectral analysis of heart rate variability. J Hypertens 6: 711-717

Kamada T, Miyake S, Kumashiro $\mathrm{M}$, Monou $\mathrm{H}$, Inoue $\mathrm{K}$ (1992) Power spectral analysis of heart rate variability in Type As and Type Bs during mental workload. Psychosom Med 54: 462-470

Keiger R, Miller JP, Bigger JT Jr, Moss A (1987) Decreased heart rate variability and its association with increased mortality after acute myocardial infarction. Am J Cardiol 59: 256-262

Kohara K, Hara-Nakamura N, Hiwada K (1995) Left ventricular mass index negatively correlates with heart rate variability in essential hypertension. Am J Hypertens 6: 183-188

Kripke DF, Mullaney DJ, Messin S, Wyborney VG (1978) Wrist actigraphic measures of sleep and rhythms. Electroencephalo Clin Neurophysiol 11: 674-676

Kupari M, Virolainen J, Koskinen P, Tikkanen MJ (1993) 
Short-term heart rate variability and factors modifying the risk of coronary artery disease in a population sample. Am J Cardiol 72: 897-903

Mandawat MK, Wallbridge DR, Pringle SD, Riyami AAS, Shahid L, Macfarlane PW, Lorimer AR, Cobbe SM (1995) Heart rate variability in left ventricular hypertrophy. $\mathrm{Br}$ Heart J 73: 139-144

Nakajima K, Osa A (1996) Detection of physiological parameters without any physical constraints in bed using sequential imaging processing. Jpn J Appl Phys 35: 269-272 [In Japanese]

Sharpley CF, Kamen P, Galatsis M, Heppel R, Veivers C, Claus $\mathrm{K}$ (2000) An examination of the relationship between resting heart rate variability and heart rate reactivity to a mental arithmetic stressor. Appl Psychophysiol Biofeedback 25: 143-153

Tanaka S (2000) Unconstrained and noninvasive automatic measurement of respiration and heart rates using a strain gauge. Transactions of the Society of Instrument and Control Engineering 30: 227-233 [In Japanese]

Tomek I (1975) Ballistocardiography: principles, simulation, applications. Adv Cardiovasc Phys 2: 119-157

Watanabe H, Watanabe K (1999) Non-invasive sensing of ballistocardiograms, respiration, snoring, body movement and coughing of a patient on the bed. Trans Soc Instrument Control Eng 35: 1012-1019 [In Japanese]

Wheeler T, Watkins PJ (1973) Cardiac denervation in diabetes. Br Med J 4: 584-586

Received: May 24, 2005

Accepted: June 13, 2006

Correspondence to: Shima Okada, Division of Health Sciences, Osaka University Graduate School of Medicine, 1-7 Yamadaoka Suita-shi, Osaka 565-0871, Japan

Phone: +81-6-6879-2526

e-mail: shima@sahs.med.osaka-u.ac.jp 